\title{
Coronal Mass Ejection deflection in the corona during the two last solar minima
}

\author{
Fernando Marcelo López ${ }^{1}$, Hebe Cremades ${ }^{2}$ and Laura Balmaceda ${ }^{3}$ \\ ${ }^{1}$ Universidad Nacional de San Juan - FCEFyN \\ Av. Ignacio de la Roza 590 (O), J5402DCS, San Juan, Argentina \\ email: fermlop@gmail.com \\ ${ }^{2}$ UTN - Facultad Regional Mendoza/CONICET \\ Rodriguez 243, Ciudad, Mendoza M5502AJE, Argentina \\ email: hebe.cremades@frm.utn.edu.ar \\ ${ }^{3}$ Instituto de Ciencias Astronómicas, de la Tierra y el Espacio/CONICET \\ Av. España Sur 1512, J5402DSP, San Juan, Argentina \\ email: labalmaceda@gmail.com
}

\begin{abstract}
In the framework of the IAU Working Group on Comparative Solar Minima, we investigate the latitudinal deflection of Coronal Mass Ejections (CMEs) with respect to the location of their uniquely identified solar source regions. Data compiled during the Whole Sun Month (WSM) and Whole Heliosphere Interval (WHI) campaigns allowed for comparisons between the two last solar minima.

The analysis of the coronal streamers' distribution during these intervals led to study of the dependence of CME deflection on the angular separation between their source regions and the nearest streamer. All performed analyses consider exclusively projected structures on the plane of the sky, disregarding longitudinal deflections as well.

The results of the present study indicate that for both minima most of the events $(62.5 \%$ for WSM, $84.2 \%$ for WHI) are deflected towards the nearest streamer, following the boundary conditions imposed by the heliospheric current sheet.

Most of the deflections found in the WHI period could be explained by the more complex structure in the global distribution of magnetic field present during that minimum. On the other hand, the low number of events detected during the WSM period hinders the statistical comparison between both campaigns.
\end{abstract}

Keywords. Sun: corona, Sun: coronal mass ejections (CMEs), Sun: activity

\section{Introduction}

Coronal Mass Ejections (CMEs) are known to be an important source of geomagnetic storms, which makes the study of their direction of propagation in the corona and interplanetary medium crucial, in order to determine the potential arrival of events to the terrestrial magnetosphere. Therefore, the deflection suffered by CMEs in the corona is one of the fundamental parameters to be considered at the time of forecasting a potentially geoefective event.

The deflection of CMEs in the solar corona and its relationship with coronal features have been previously studied. Cremades et al. (2006) found a connection between CME deflection and coronal hole (CH) location and area. Similarly, Gopalswamy et al. (2009) found that CMEs are significantly affected when the eruptions occur close to a $\mathrm{CH}$, with the $\mathrm{CH}$ acting as a magnetic wall constraining the CME trajectory. Not in conflict with these results, Gui et al. (2011) suggest that CMEs tend to deflect to regions with lower magnetic energy density. Recent detailed case studies aimed to understand the 
fundamentals of CME deflection include those of Shen et al. (2011) and Zuccarello et al. (2012). In view of hitherto existing evidence of the influence of the corona's environment on early CME trajectory, and the essentially different coronal magnetic configuration present during the past two solar minima, this report deals with CME deflections during a full rotation of these minima and their connection to streamers, white-light coronal features that denote global magnetic field configuration.

The next Section describes the event selection process, followed by the main results about the types of CME-streamer interaction and the relationship between CME deflection and distance to streamer. Main findings are discussed and summarized in Section 5.

\section{Events Selection}

The WSM (10 August to 8 September 1996) and the WHI (20 March to 16 April 2008) campaigns represent a unique opportunity to compare different aspects of the corona/heliosphere during the last two solar magnetic minima. Using data from the Solar and Heliospheric Observatory (SOHO), Yohkoh, and Solar-Terrestrial Relations Observatory (STEREO), Cremades et al. (2011) identified all distinguishable coronal transients during these time intervals, from wide CMEs up to very narrow jets. Here, we analyze a subset of those events fulfilling the following criteria: 1) their angular width is higher than $10^{\circ}$, and 2) their solar source region can be uniquely identified on images of the Extreme-UV Imaging Telescope (SOHO/EIT) or Extreme UltraViolet Imager (STEREO/EUVI). Following these criteria, the original set of events reduced to a total of 8 events for WSM and 19 for WHI. Furthermore, this analysis considers only streamers and disregards pseudostreamers. The first separate open field lines of opposite polarities and are thus associated with the heliospheric current sheet (HCS), while the latter overlie loop arcades separating field lines of the same polarity.

\section{Types of CME - Streamer Interaction}

When inspecting the interaction between CMEs and streamers, we found three main classes, according to their behaviour on images of the corona and Carrington maps from the Large Angle and Spectrometric Coronagraph (LASCO C2). The kinds of interaction we found are as follows: 1) "Regular Case", when there is a well-defined, narrow streamer and the CME is not "channeled" through it; 2) "Channeled CME", when the CME travels

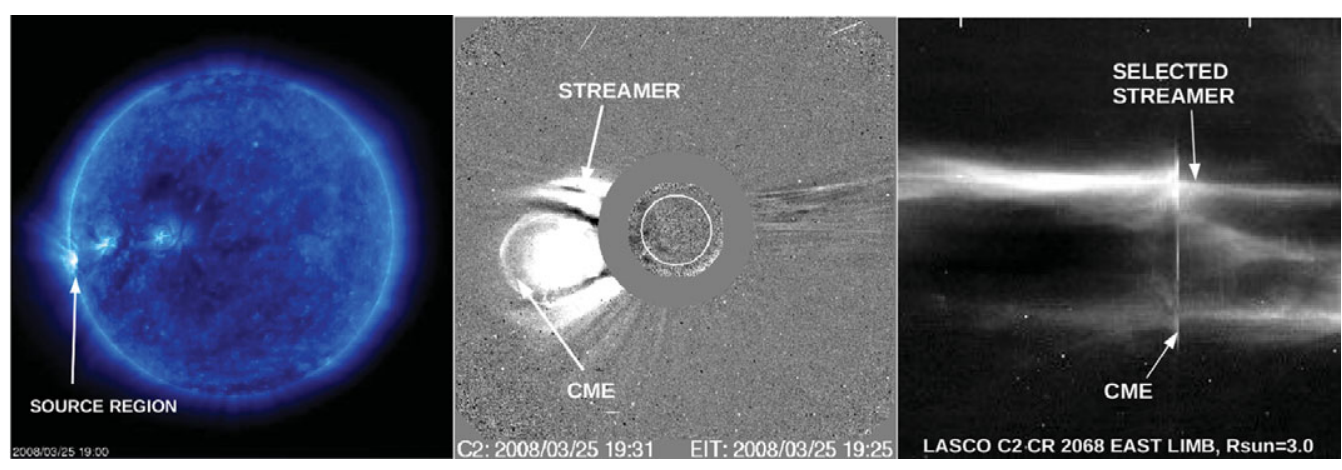

Figure 1. Example of the kind of interaction "Regular Case": EUV image with the associated source region (left), LASCO C2 running difference image (centre), and the corresponding LASCO C2 Carrington map obtained at 3 solar radii from the Sun's centre (right). 
within the streamer, i.e. in the direction determined approximately by the central PA of the streamer; and 3) "Spread-Out Streamer" when the streamer is diffuse and wide.

Fig. 1 presents an example of the first kind of interaction mentioned above. To the left, an EUV image shows the associated source region, while the ensuing CME can be appreciated in a LASCO C2 running difference image (centre). The corresponding LASCO C2 Carrington map taken at 3 solar radii from the Sun's centre allows the visualization of the streamers' behaviour in time (right).

\section{Relationship Between CME Deflection and Distance to Streamers}

After classifying the events according to the three types defined above, we study the dependence of CME deflection on the angular distance between the associated source region
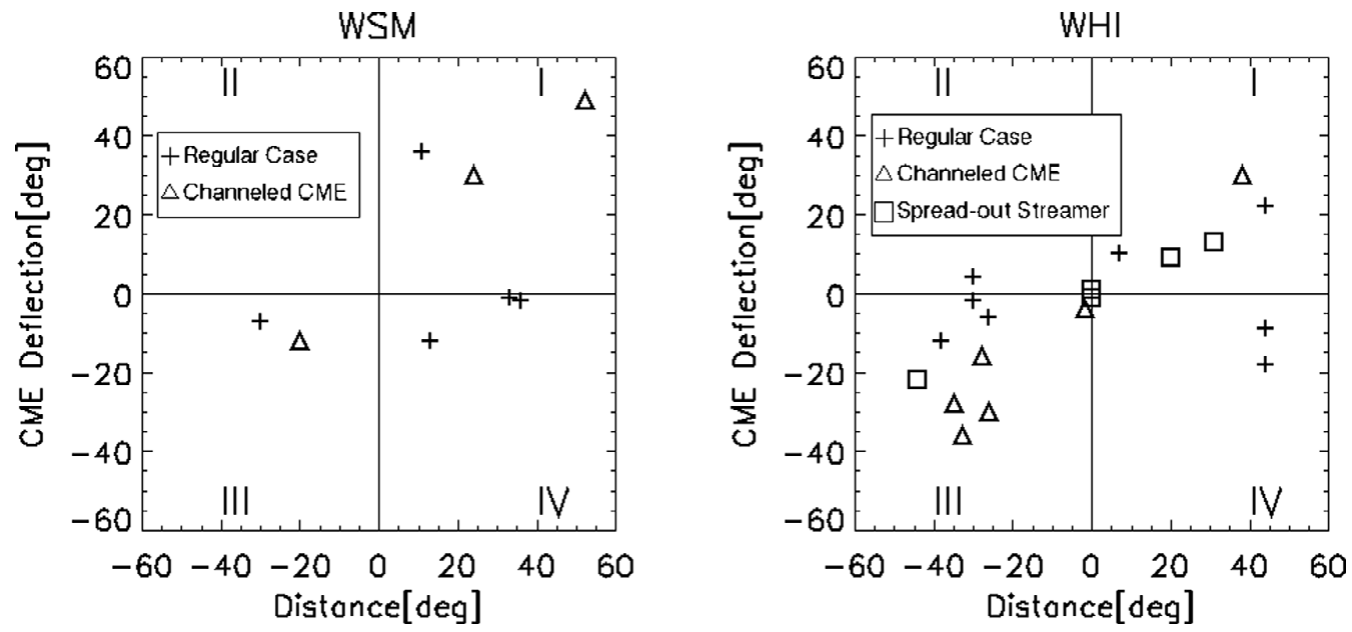

Figure 2. Relationship between the deflection of CMEs and the distance from their source region during the WSM (left) and WHI (right).

and the nearest streamer. This angular distance is given by the difference of the respective position angles, i.e. distance=ST_PA - SR_PA. The CME deflection angle is given by the difference between those position angles of the CME and its associated source region, i.e. CME deflection=CME_PA - SR_PA). The CME central position angles were obtained from the SOHO/LASCO's catalog of CMEs (http://cdaw.gsfc.nasa.gov/CME_list/index.html), whereas the streamer position angles were determined using LASCO C2 Carrington maps. The source region position angles were taken from Cremades et al. (2011). Most of the low-coronal sources of the events here analyzed were located at heliographic longitudes higher than $50^{\circ}$. With the source regions close to the solar limb, projection effects are kept to a minimum.

Fig. 2 compares WSM and WHI with regard to CME deflection as a function of the angular distance between source regions and streamers. The three different types of interaction are represented by different symbols. In order to analyze the direction of CME deflection, we divide the plots in Fig. 2 into quadrants (I, II, III and IV) centred at distance $=0$ and $\mathrm{CME}$ deflection $=0$. When the deflection and distance angles are of the same sign, the CME deflects towards the streamer (quadrants I and III in Fig. 2). On the other hand, if the signs are opposite, the CME deflects in the direction opposite to that of the streamer (quadrants II and IV in Fig. 2). It is evident from the figure that the general behaviour follows these rules. In order to quantify the goodness of the linear 
Table 1. Correlation coefficients between the CME deflection and distance angles during WSM and WHI for the three types of interaction.

\begin{tabular}{lccc}
\hline & Regular Case & Channeled CME & Spread-Out Streamer \\
WSM & $\mathrm{r}=0.08$ & $\mathrm{r}=0.99$ & - \\
WHI & $\mathrm{r}=0.12$ & $\mathrm{r}=0.96$ & $\mathrm{r}=0.97$ \\
\hline
\end{tabular}

relationship between CME deflection and distance angles, we calculated the correlation coefficient (r) for each of the three types of interaction (see Table 1).

\section{Discussion}

From the definitions of the CME deflection and distance angles, it arises that CME deflections away from their source regions towards the nearest streamer and the distance angle must have the same sign. On the contrary, the signs are opposite when deflections are such that the CME moves away from the streamer.

Most of the events here analyzed (16 out of 19 , i.e. $84.2 \%$ for WHI; and 5 out of 8, i.e. $62.5 \%$ for WSM) are indeed deflected towards the nearest streamer, following the boundary conditions imposed by the HCS. This occurs in spite of the essentially different configuration of the HCS during both of the inspected minima.

From the correlation analysis it is found for both time intervals, that those events classified as "Regular Case" do not present a linear relationship between the deflection and distance angles, even showing deflections in the direction opposite to the streamer. On the other hand, events classified as "Channeled CME" or "Spread-Out Streamer" present very good correlation coefficients, with all deflections happening in the streamer direction.

The high amount of deflections towards the nearest streamer is especially evident for the WHI period. For the WSM, the relationship between both angles follows the same trend, though with higher scattering. It is very likely that the lack of events during the WSM period is hindering the analysis.

The different coronal structure exhibited by the Sun during the two analyzed periods of minimum yielded also different absolute CME deflections. However, this is logical given the essentially distinct configuration of the HCS and the solar corona. Still, during both periods most CMEs deflected towards their respective nearest streamer, showing a similar behavior and thus indicating the same physical principle in action.

\section{Acknowledgements}

The authors acknowledge useful discussions during the Symposium with F. Zuccarello. L.B. and H.C. are members of the Carrera del Investigador Científico of CONICET.

\section{References}

Cremades, H., Bothmer, V., \& Tripathi, D. 2006, Adv. Space Res, 38, 461.

Cremades, H., Mandrini, C. H., \& Dasso, S. 2011, Solar Phys., 274, 233.

Gopalswamy, N., Mäkelä, P., Xie, H., Akiyama, S., \& Yashiro, S., 2009, J. Geophys. Res., 114, $\mathrm{A} 00 \mathrm{~A} 22$.

Gui, B., Shen, C., Wang, Y., Ye, P., Liu, J., Wang, S., \& Zhao, X., 2011, Solar Phys., 271, 111. Shen, C., Wang, Y., Gui, B., Ye, P., \& Wang, S., 2011, Solar Phys., 269, 389.

Zuccarello, F., P., Bemporad, A., Jacobs, C., Mierla, M., Poedts, S., \& Zuccarello, F., 2012, ApJ, 744, 66 . 